\title{
Synthesis and Visible-Light Photochromism of a Donor-Acceptor Type of Dimethyl 2,3-bis(arylethynyl)fumarate
}

\author{
Ryota Sakamoto
}

\begin{abstract}
Herein, the author synthesized a donor-acceptor type of photochromic dimethyl 2,3-bis(arylethynyl)fumarate, $(E)-2$, where ferrocene and 4-nitrobenzene were employed as donor and acceptor arenes, respectively. Compared to parent dimethyl 2,3-bis(ferrocenylethynyl)fumarate, $(E)-2$ enjoyed more profound donor-acceptor interaction, leading to a red-shifted intramolecular charge transfer band. $(E)-2$ underwent visible-light photochromism upon excitation with the intramolecular charge transfer band.
\end{abstract}

Index Terms-Photochromism, ferrocene, donor-acceptor interaction.

\section{INTRODUCTION}

Ethynylethene was indeed synthesized several decades ago [1], though, it is Dierderich who has put spotlight on this series of compounds in photochemical and synthetic aspects [2]-[7]. This class of compounds has an extended $\pi$-system and shows $E$ to $Z$ photoisomerization around the $\mathrm{C}=\mathrm{C}$ bond upon excitation of the $\pi-\pi^{*}$ transition. Unlike azobenzene and a portion of stilbene, the $Z$ form is thermally stable, which is one of the virtues of ethynylethene. In addition, good accessibility to a variety of substituents through the ethynyl bond is also one of the features in ethynylethene, by utilizing cross coupling reactions such as Sonogashira-Hagiwara [8], [9] and Stille reactions [10]. Dierderich and coworkers have synthesized a wide range of ethynylethene derivatives, to realize a three-dimensional photoswitching system [3], and emitters with high quantum yields [4].
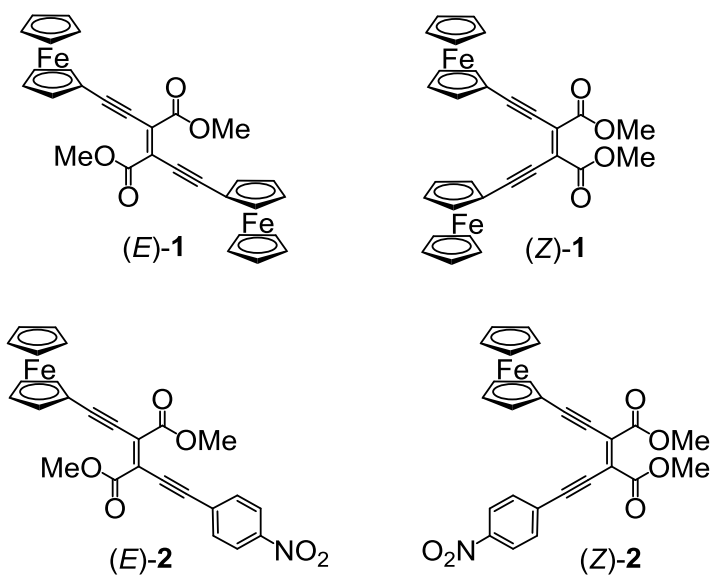

Fig. 1. Molecular structures of $\mathbf{1}$ and $\mathbf{2}$

Manuscript received April 7, 2015; revised May 25, 2015.

Ryota Sakamoto is with the University of Tokyo, Japan (e-mail: sakamoto@chem.s.u-tokyo.ac.jp).
We have found a new class of diethynylethene frameworks, dimethyl 2,3-bis(arylethynyl)fumarate/maleate, to be an excellent photochromic and acceptor fragment [11], [12]. In combination with electron-donating ferrocene, unique system $(E)-1, \quad$ or dimethyl 2,3-bis(ferrocenylethynyl)fumarate/maleate (Fig. 1), was established to produce an optical switch in the electronic communication with photoisomerization triggered by the excitation of a intramolecular charge transfer (ICT) band in the visible region [11], [12].

Herein, the author synthesizes anew a donor-acceptor type of dimethyl 2,3-bis(arylethynyl)fumarate, $(E)-2$, where ferrocene and 4-nitrobenzene are employed as donor and acceptor arenes, respectively (see Fig. 1). Its donor-accepter interaction and visible-light photochromism are investigated.

\section{EXPERIMENTAL}

\section{A. Materials}

Ethynylferrocene, [11] dimethyl 2,3-dibromofumarate,[12] dimethyl 2,3-bis(ferrocenylethynyl)fumarate, $(E)$-1,[Angew] were prepared according to the literature procedures. Trimethylamine $\left(\mathrm{Et}_{3} \mathrm{~N}\right)$ was distilled with $\mathrm{KOH}$ tablets and stored with $\mathrm{KOH}$ tablets. THF was stored under $\mathrm{N}_{2}$ atmosphere without any stabilizer and distilled with metal $\mathrm{Na}$ and benzophenone just before reactions. 1,4-Dioxane (Dehydrated grade) was further dried with activated molecular sieves (3A). The other materials were used as purchased. $\mathrm{CuI}$ was provided by Wako Pure Chemical Industries, Ltd. $\mathrm{PdCl}_{2}\left(\mathrm{PPh}_{3}\right)_{2}$ and 4-iodonitrobenzene were supplied by Tokyo Chemical Industry Co., Ltd. The other chemicals were supplied by Kanto Chemical Co., Inc.

\section{B. Syntheses of \\ 1-(4-Nitrophenyl)-2-triisopropylsilyllacethylene}

4-Iodonitrobenzene (3.0g, $12 \mathrm{mmol}), \mathrm{PdCl}_{2}\left(\mathrm{PPh}_{3}\right)_{2}(96$ $\mathrm{mg}$ ), and $\mathrm{CuI}(94 \mathrm{mg})$ were suspended in THF (140 mL) under a nitrogen atmosphere. To the suspension triisopropylsilylacethylene $(5 \mathrm{~mL}, 22 \mathrm{mmol})$ and $\mathrm{Et}_{3} \mathrm{~N}(60 \mathrm{~mL})$ were added. The dark-orange solution was then refluxed for $14 \mathrm{~h}$. The reaction mixture was filtrated through celite, and after evaporation the residue was purified with column chromatography $\left(\mathrm{Al}_{2} \mathrm{O}_{3}\right.$, activity II-III) with a mixture of hexane and dichloromethane (3:1 v/v) as eluent The yellow fraction was collected, evaporated, and then recrystallized from dichloromethane and hexane to give pale brown solid of 1-(4-nitrophenyl)-2-triisopropylsilyllacethylene: yield; $3.0 \mathrm{~g}$ 
(82\%). ${ }^{1} \mathrm{H}-\mathrm{NMR}\left(\right.$ Chloroform- $\left.d_{1}\right): \delta 8.15(\mathrm{~d},(8.9), 2 \mathrm{H}), 7.58$ (d, (8.9), 2H), 1.15-1.05 (m, 21H).

\section{Synthesis of 4-Nitrophenylacethylene}

1-(4-Nitrophenyl)-2-triisopropylsilyllacethylene (3.0g, 9.9 mmol) was dissolved in THF $(200 \mathrm{~mL})$ under a nitrogen atmosphere. To the solution ${ }^{n} \mathrm{Bu}_{4} \mathrm{NF}$ in THF (with $5 \mathrm{w} \% \mathrm{H}_{2} \mathrm{O}$ ) $(12 \mathrm{ml}, 12 \mathrm{mmol})$ was added dropwise in five minutes, which afforded color change from brown to dark blue. After stirring $5 \mathrm{~min}$, to the solution ether and water was added, and the organic phase was separated and washed with water and brine Dried over $\mathrm{Na}_{2} \mathrm{SO}_{4}$, the solution was evaporated and residue was purified with alumina column chromatography (activity II-III) with ether as eluent. The yellow fraction was collected and evaporated, and the residue was recrystallized from dichloromethane and hexane to give pale brown soft solid of 4-nitrophenylacethylene: yield; $1.1 \mathrm{~g}(76 \%)$.

\section{Synthesis of (E)-Dimethyl \\ 2-(ferrocenylethynyl)-3-(4-nitrophenylethynyl)fumarate, (E)-2}

To dimethyl 2,3-dibromofumarate (443 $\mathrm{mg}, 1.5 \mathrm{mmol}$ ), $\mathrm{CuI}$ (14 mg), $\mathrm{PdCl}_{2}\left(\mathrm{PPh}_{3}\right)_{2}$ (12 mg), 4-nitroethynylbenzene (210 $\mathrm{mg}, 1.4 \mathrm{mmol}$ ), and ethynylferrocene $(300 \mathrm{mg}, 1.4$ $\mathrm{mmol})$, dehydrated $\mathrm{Et}_{3} \mathrm{~N}(50 \mathrm{~mL})$ were added under a nitrogen atmosphere. The brown suspension was heated at $100^{\circ} \mathrm{C}$, which brought it into red suspension. After refluxing $2 \mathrm{~h}$, to the red suspension dichloromethane was added, and the red solution was filtered through celite. After removing the solvents black-red residue was purified by alumina (activity II-II) column chromatography with hexane-dichloromethane $(1: 1 \mathrm{v} / \mathrm{v})$ as eluent. The deep-red fraction was evaporated to obtain $(E)-\mathbf{2}$ as deep-red solid: yield; $29 \mathrm{mg}$ (4.1\%). Recrystallization from dichloromethane and hexane in dark afforded deep-red crystals of $(E)$-2. ${ }^{1} \mathrm{H}-\mathrm{NMR}$ (Toluene- $\left.d_{8}\right): \delta$ 7.62 (d, (8.8), 2H), 4.38 (dd, (1.9, 1.9), 2H), 3.98-3.97 (m, $7 \mathrm{H}), 3.50(\mathrm{~s}, 3 \mathrm{H}), 3.41(\mathrm{~s}, 3 \mathrm{H})$ (Residual protons were hidden by signals derived from Toluene- $d_{8}$ ).

\section{E. UV-Vis Spectroscopy}

As a UV-Vis spectrometer, a set of V-570 (by JASCO Inc.) and $\mathrm{PC}$ as a recorder was implemented. To observe absorptions in the UV region quartz cells (optical path: $1 \mathrm{~cm}$ ) were utilized. Each solution was fabricated to have a maximum optical density around 0.5 . Dichloromethane (High Performance Liquid Chromatography grade, Kanto Chemical Co., Inc.), DMF (Spectroscopy grade, Kanto Chemical Co., Inc.), and heptane (Special grade, Kanto Chemical Co., Inc.) were used as purchased.

\section{F. DFT Calculation}

In the DFT calculation, the three-parameterized Becke-Lee-Yang-Parr (B3LYP) hybrid exchange-correlation functional was employed. The geometry of $(E)-\mathbf{1}$ was optimized by the DFT(B3LYP) method without solvent effect, using the crystal structure as the initial value. As to $(E)-\mathbf{2}$, the initial values were employed from the optimized structure of (E)-1. As a basis set Lanl2DZ (Hay-Wadt ECP) was employed for all atoms. For the comparison with the electronic spectra observed in dichloromethane, the solvent effect was considered with the polarized continuum model
(PCM) in the TD-DFT method. A series of calculation mentioned here is implemented with Gaussian 03 or Gaussian 03W (revision-B.05) [15].

\section{G. Tracking of the Photoisomerization Behavior}

As an NMR spectrometer a set of Bruker $500 \mathrm{MHz}$ and PC as a recorder was implemented. To irradiate the UV light directly, NMR tubes made of quartz were utilized. As a deuterized solvent, toluene- $d_{8}$ (Ample-sealed, Sigma-Aldrich Co.) was used as purchased. To avoid the evaporation of the solvent the sample tubes were tightly sealed with rubber septums, Para film, and Teflon tape. As a photon source an $\mathrm{Hg}$ high pressure lamp (Ushio Inc.) was implemented, assorting certain bright lines with a monochromator (CT-10T, JASCO Inc.), or visible cut-off, IR-cut, and UV transmission filters (Asahi Techno Glass Inc.).

\section{UV-VIS SPECTROSCOPY}

The exploration of the nature of a charge transfer band and solvatochromism on that greatly correlates with the ability as second-order non-linear optics (NLOs), though there are other parameters to be considered, such as the molecular and crystallographic symmetry, for actual application.

In the two-level model [13] the relationship between the ability and nature of an electronic band is given as

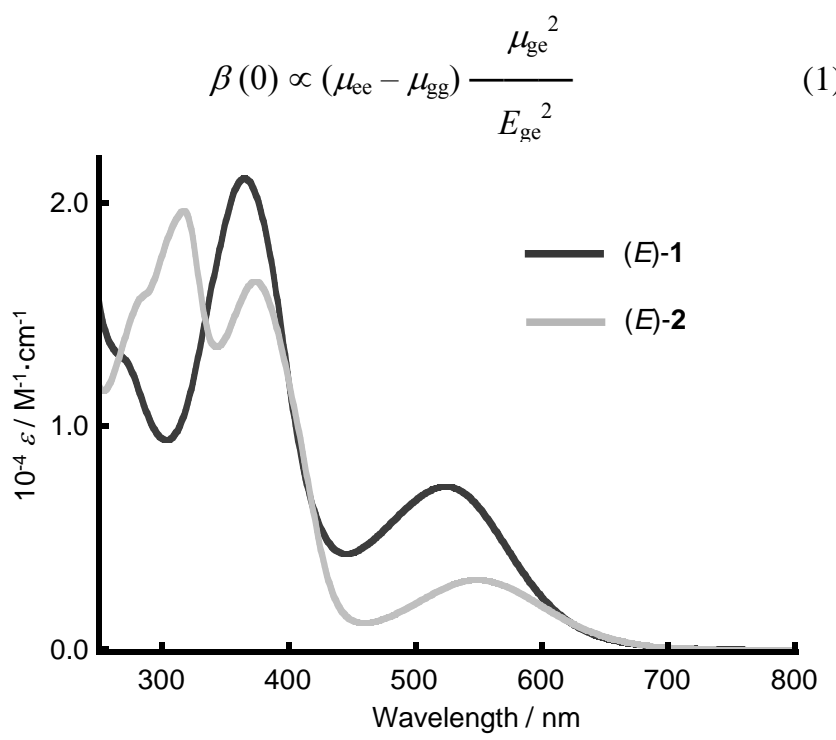

Fig. 2. UV-Vis spectra of $(E)-\mathbf{1}$ and $(E)-\mathbf{2}$ in dichloromethane.
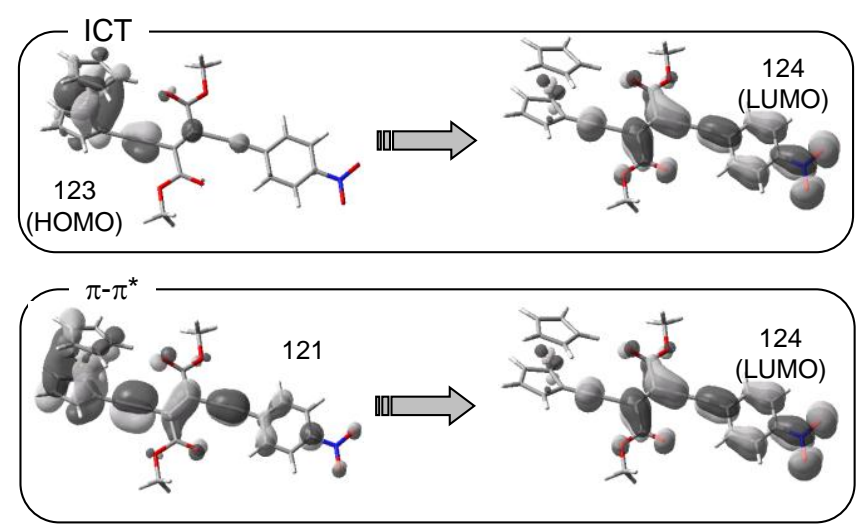

Fig. 3. Main transitions in the ICT and $\pi-\pi^{*}$ band of $(E)$-2. 


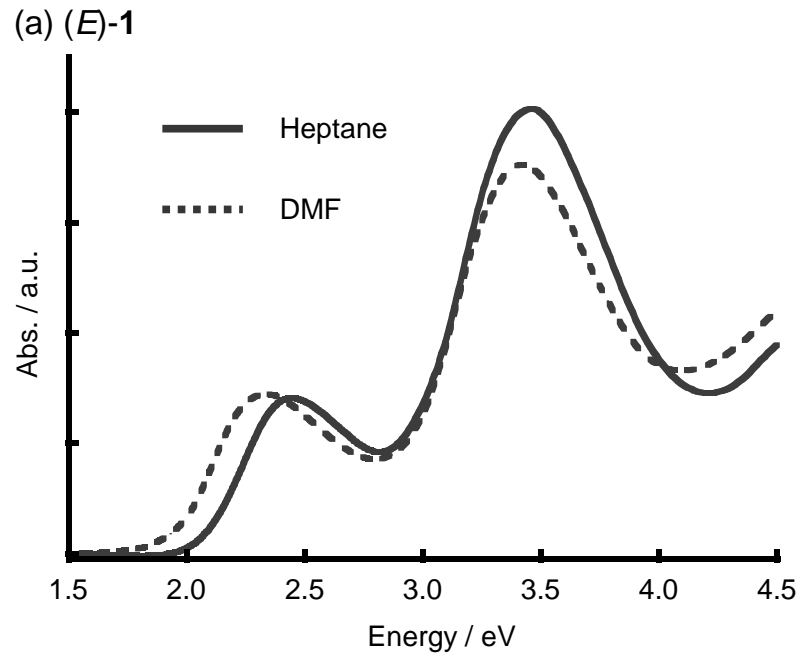

(b) $(E)-2$

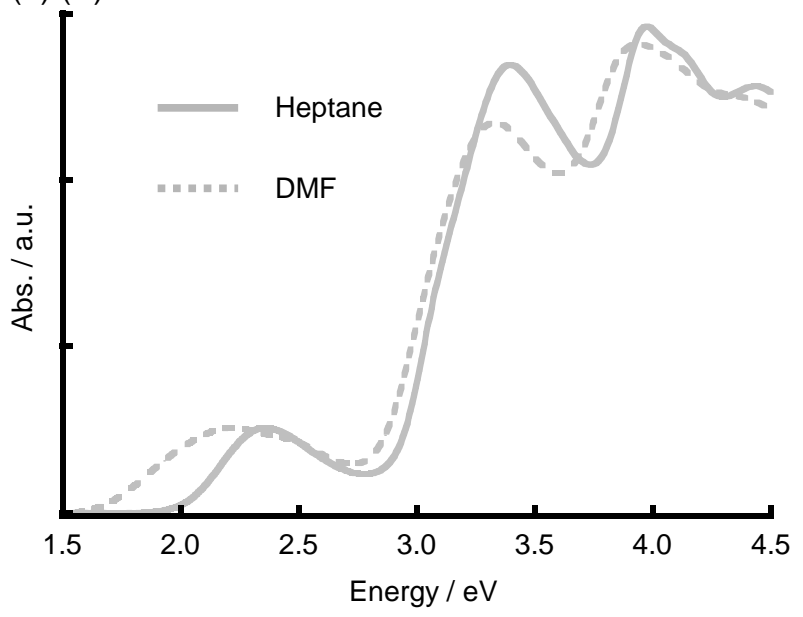

Fig. 4. UV-Vis spectra of (E)-1 and (E)-2 in hexane and DMF .

TABLE I: OPTICAL PROPERTIES OF $(E)-\mathbf{1}$ AND $(E)$-2

\begin{tabular}{|c|c|c|c|}
\hline Compound & Solvent & $\pi-\pi^{*} / \mathrm{eV}(\mathrm{nm})$ & $\mathrm{ICT} / \mathrm{eV}(\mathrm{nm})$ \\
\hline$(E)-\mathbf{1}$ & DMF & $3.42(363)$ & $2.33(532)$ \\
\hline & Dichloromethane & $3.40(365)$ & $2.37(524)$ \\
\hline & Heptane & $3.45(359)$ & $2.44(508)$ \\
\hline$(E)-\mathbf{2}$ & DMF & $3.33(372)$ & $2.21(559)$ \\
\hline & Dichloromethane & $3.32(374)$ & $2.26(548)$ \\
\hline & Heptane & $3.40(365)$ & $2.36(526)$ \\
\hline
\end{tabular}

where $\beta(0)$ denotes the static molecular hyperpolarizability, which correlates with the second-order optical ability, $\mu_{\mathrm{ee}}$ and $\mu_{\mathrm{gg}}$ indicate the molecular dipole moment in the excited and ground states, and $\mu_{\mathrm{ge}}$ and $E_{\mathrm{ge}}$ are the transition dipole moment and transition energy of the electronic band, respectively. This equation implies that compounds bearing the larger absorption $\left(\mu_{\mathrm{ge}}\right)$ with the longer wavelength maximum (Ege), and the greater difference in the molecular dipole moment between the excited and ground state $\left(\mu_{\mathrm{ee}}\right.$ $\mu_{\mathrm{gg}}$ ), are more favorable as second-order NLOs. Compounds with charge transfer bands suit this condition pretty well. Such compounds tend to show intense solvatochromism due to the large change in $\left(\mu_{\mathrm{ee}}-\mu_{\mathrm{gg}}\right)$ that accompanies the optical electron transfer, hence the extent of the solvatochromism can be one of the most accessible indicators.

Fig. 2 shows the electronic spectra of $(E)-\mathbf{1}$ and $(E)-\mathbf{2}$ in dichloromethane, and Table 1 enumerates their parameters. As the author reported previously, the two distinctive absorption bands in (E)-1 at 365 and $524 \mathrm{~nm}$, are, respectively, assignable to the $\pi-\pi^{*}$ localized on the dimethyl 2,3-diethynylfumarate framework, and ICT transitions from ferrocene(d) to the $\pi^{*}$ of the dimethyl 2,3-diethynylfumarate framework [11],[12]. These bands are redshifted in $(E)-\mathbf{2}$, which implies that the LUMO is replaced with the unoccupied orbital mainly contributed by the 4-nitrobenzene moiety, and it is a better acceptor than the ethynylethene framework. This idea is favored with the DFT calculation (Fig. 3). It discloses that the band at $548 \mathrm{~nm}$ is assignable to ICT from ferrocene(d) to the $\pi^{*}$ orbital of the 4-nitrobenzene moiety, whereas that at $374 \mathrm{~nm}$ is fundamentally $\pi-\pi^{*}$ transition of the dimethyl 2,3-diethynylfumarate framework, with bear a slight charge transfer nature.

Fig. 4 shows the normalized electronic spectra of $(E)-\mathbf{1}$ and $(E)-2$ in heptane and DMF, and Table 1 displays the solvatochromic shift of the absorption maxima on both $\pi-\pi^{*}$ and ICT bands. (E)-2 showed larger redshifts as to both bands than those of $(E)-1$. In addition, in both compounds the ICT band exhibited a larger bathochromic shift than the $\pi-\pi^{*}$ band. This series of facts ensures that the lowest-lying electronic bands in $(E)$-1 and $(E)$-2 are charge transfer bands, and $(E)-\mathbf{2}$ may be a potential second-order NLO material.

\section{PHOTOISOMERIZATION}

Upon irradiation with 546 and $578 \mathrm{~nm}$ visible light, $(E)-2$ in toluene- $d_{8}$ underwent ${ }^{1} \mathrm{H}$ NMR spectral change as shown in Fig. 5. This series of spectral changes are typical of $E$ to $Z$ photoisomerization in dimethyl 2,3-bis(arylethynyl)fumarate [11],[12] (Fig. 1). The conversion ratio was calculated to be $29 \%$, judging from the integral ratio of the signals of the two isomeric forms. The quantum yield for the isomerization was apparently too low to conduct a quantitative analysis.
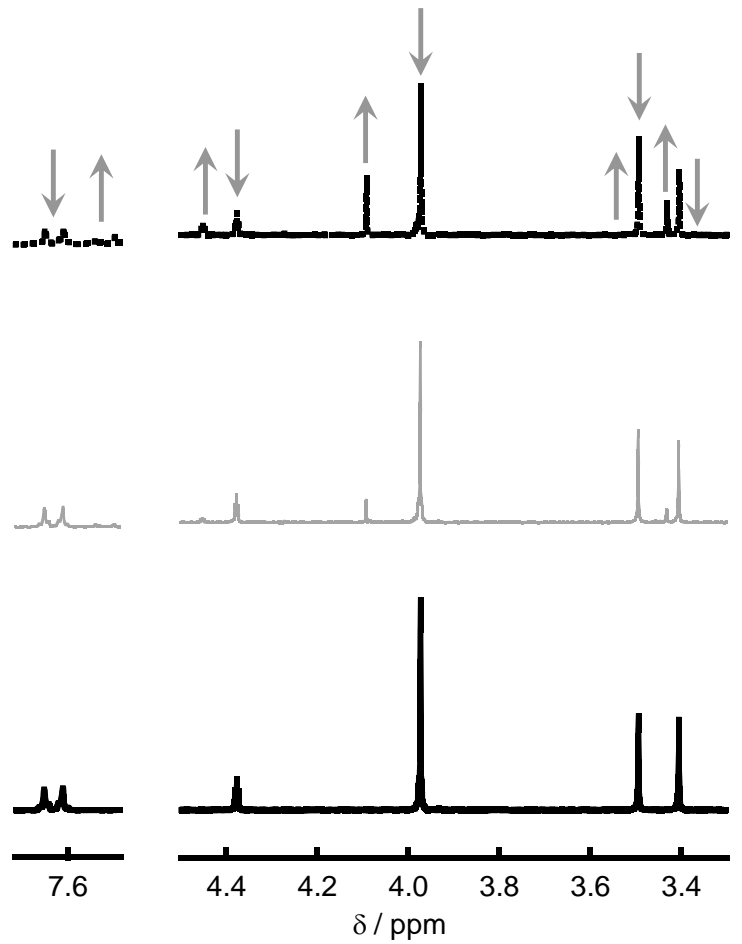

Fig. 5. Time-course ${ }^{1} \mathrm{H}-\mathrm{NMR}$ spectral changes of (E)-2 upon irradiation with light at 546 and $578 \mathrm{~nm}$ in toluene- $d_{8}$. 


\section{CONCLUSION}

The author synthesized a donor-acceptor type of photochromic dimethyl 2,3-bis(arylethynyl)fumarate, (E)-2, where ferrocene and 4-nitrobenzene were employed as donor and acceptor arenes, respectively. Compared to parent $(E)-\mathbf{1}$, $(E)-2$ enjoyed more profound donor-acceptor interaction, leading to a red-shifted ICT band, and is potentially a second-order NLO material. (E)-2 underwent visible-light photochromism to $(Z)-2$ upon excitation with the ICT band.

\section{REFERENCES}

[1] Y. Hori, N. Noda, S. Kobayashi, and H. Taniguchi, "Synthesis and properties of tetrakis(phenylethynyl)ethylene," Tetrahedron Lett., vol. 10, pp. 3563-3566, 1969.

[2] L. Gobbi, P. Seiler, F. Dierderich, and V. Gramlich, "Molecular Switching: A Fully Reversible, Optically Active Photochemical Switch Based on a Tetraethynylethene-1,1'-Binaphthalene Hybrid System," Helv. Chim. Acta., vol. 83, pp. 1711-1723, 2000.

[3] L. Gobbi, P. Seiler, and F. Diederich, "A novel three-way chromophoric molecular switch: $\mathrm{pH}$ and light controllable switching cycles," Angew. Chem., Int. Ed. vol. 38, pp. 674-678, 1999.

[4] M. J. Edelmann, J.-M. Raimundo, N. F. Utesch, and F. Diederich, "Dramatically enhanced fluorescence of heteroaromatic chromophores upon insertion as spacers into oligo(triacetylne)s," Helv. Chim. Acta. vol. 83, pp. 2195-2293, 2002.

[5] M. B. Nielsen, M. Schreiber, Y. G. Baek, P. Seiler, S. Lecomte, C. Boudon, R. R. Tykwinski, J.-P. Gisselbrecht, V. Gramlich, P. J. Skinner, C. Bosshard, P. Günter, M. Gross, and F. Diederich, "Highly functionalized dimeric tetraethynylethenes and expanded radialenes: Strong evidence for macrocyclic cross-conjugation," Chem. Eur. J., vol. 7, pp. 3263-3280, 2001.

[6] R. E. Martin, T. Mäder, and F. Diederich, "Monodisperse poly(triacetylene) rods: Synthesis of a $11.9 \mathrm{~nm}$ long molecular wire and direct determination of the effective conjugation length by UV/Vis and raman spectroscopies," Angew. Chem. Int. Ed. vol. 38, pp. 817-821, 1999.

[7] R. E. Martin, J. Bartek, Diederich, R. R. Tykwinski, E. C. Meister, A. Hilger, and H. P. Lüthi, "Photochemical trans-cis isomerisation of donor/acceptor-substituted

(E)-hex-3-ene-1,5-diynes (1,2-diethynylethenes, DEEs) and 3,4-diethynylhex-3-ene-1,5-diynes (tetraethynylethenes, TEEs)," J. Chem. Soc. Perkin Trans., vol. 2, pp. 233-242, 1998.

[8] K. Sonogashira, "Development of $\mathrm{Pd}-\mathrm{Cu}$ catalyzed cross-coupling of terminal acetylenes with $\mathrm{sp}^{2}$-carbon halides," J. Organomet. Chem., vol. 653, pp. 46-49, 2002.

[9] K. Sonogashira, Y. Tohda, and N. Hagihara, “ A convenient synthesis of acetylenes: catalytic substitutions of acetylenic hydrogen with bromoalkenes, iodoarenes and bromopyridines," Tetrahedron Lett., vol. 15 , pp. 4467-4470, 1975.

[10] D. Milstein and J. K. Stille, "A general, selective, and facile method for ketone synthesis from acid chlorides and organotin compounds catalyzed by palladium, “ J. Am. Chem. Soc., vol. 100, pp. 3636-3638, 1978.

[11] R. Sakamoto, M. Murata, and H. Nishihara, "Visible light photochromism of bis(ferrocenylethynyl)ethenes to switch electronic communication between ferrocene sites," Angew. Chem. Int. Ed., vol. 45, pp. 4793-4795, 2006.

[12] R. Sakamoto, S. Kume, and H. Nishihara, "Visible-light photochromism of triarylamine- or ferrocene-bound diethynylethenes that switches electronic communication between redox sites and luminescence," Chem. Eur. J., vol. 14, pp. 6978-6986, 2008.

[13] J. L. Oudar and D. S. Chemla, "Hyperpolarizabilities of the nitroanilines and their relations to the excited state dipole moment," $J$. Chem. Phys., vol. 66, pp. 2664-2668, 1977.

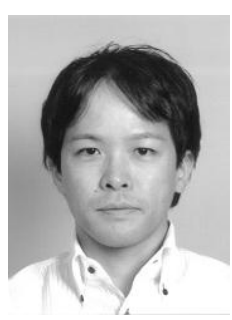

Ryota Sakamoto was born in Yamagata Vil., Nagano, Japan, in 1980. He graduated from the University of Tokyo, Japan in 2002, and received his $\mathrm{PhD}$ degree from the same university in 2007 under the supervision of Prof. Hiroshi Nishihara.

Then he was appointed as an assistant professor at the Tokyo University of Science (Japan), working with Prof. Takeshi Yamamura. In 2010 he moved to the University of Tokyo, joining Prof. Nishihara's group again. His current research interest lies in the construction of molecule-based nanostructures, and photonic and electronic devices using them. 